\title{
Erratum to: Unexpected transitional paths in the prolate to oblate shape phase transitions for Bose-Fermi systems
}

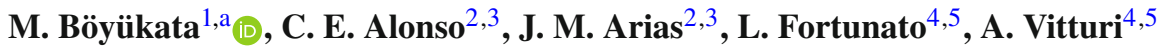 \\ ${ }^{1}$ Physics Department, Faculty of Science and Arts, University of Kırıkkale, 71450 Kırıkkale, Turkey \\ ${ }^{2}$ Departamento de Física Atómica, Molecular y Nuclear, Facultad de Física, Universidad de Sevilla, Apartado 1065, 41080 Sevilla, Spain \\ ${ }^{3}$ Instituto Carlos I de Física Teórica y Computacional, Universidad de Granada, Fuentenueva s/n, 18071 Granada, Spain \\ ${ }^{4}$ Dipartimento di Fisica e Astronomia "G. Galilei”, Università di Padova, Padova, Italy \\ ${ }^{5}$ INFN, Sezione di Padova, Via Marzolo 8, 35131 Padova, Italy
}

Received: 16 September 2021 / Accepted: 17 September 2021 / Published online: 19 October 2021

(C) The Author(s), under exclusive licence to Società Italiana di Fisica and Springer-Verlag GmbH Germany, part of Springer Nature 2021

Erratum to: Eur. Phys. J. A (2021) 57:2

https://doi.org/10.1140/epja/s10050-020-00308-4

This work was supported by the Scientific and Technical Research Council of Turkey (TÜBİTAK), under the project number 119F127, by the Consejería de Economía, Conocimiento, Empresas y Universidad de la Junta de Andalucía (Spain) under Group FQM-160, by the Spanish
Ministerio de Ciencia e Innovacióon, ref. FIS2017-88410-P and PID2019-104002GB-C22, and by the European Commission, ref. H2020-INFRAIA-2014-2015 (ENSAR2). In the acknowledgments part of the published version of Eur. Phys. J. A 57 (2021) 2, the project number is corrected as 119F127. The original article can be found online at https:// doi.org/10.1140/epja/s10050-020-00308-4.

The original article can be found online at https://doi.org/10.1140/ epja/s10050-020-00308-4.

a e-mail: boyukata@kku.edu.tr (corresponding author) 